\title{
Mesoscopic versus Macroscopic division of current fluctuations.
}

\author{
V. Rychkov* and M. Büttiker \\ Département de Physique Théorique, Université de Genève, CH-1211 Genève 4, Switzerland
}

(Dated: September 29, 2018)

\begin{abstract}
We investigate the current shot noise at a three terminal node in which one of the branches contains a noise generating source and the correlations are measured between the currents flowing through the other two branches. Interestingly, if the node is macroscopic, the current correlations are positive, whereas for a quantum coherent mesoscopic node anti-bunching of electrons leads to negative correlations. We present specific predictions which permit the experimental investigation of the crossover from quantum mechanical noise division to macroscopic noise noise division.
\end{abstract}

PACS numbers: 73.23.b, 72.70.+m, 03.65.Yz

Introduction - Over the past two decades the theoretical and experimental investigation of the noise properties of small conductors has developed into a major field of research in mesoscopic physics. Fundamentally shot noise is a consequence of the granularity of charge and quantum diffraction [1]. In quantum coherent conductors shot noise arises whenever there are multiple final states for a given incident state. For purely elastic scattering, for conductors embedded in a zero-impedance external circuit, the Pauli exclusion principle leads to negative current correlations independent of the shape and form of the conductor [2]. Negative current correlations have been measured at beam splitters and in quantum Hall effect geometries [3, 4, [5]. If the occupation of the incident channel is small and approaches a Maxwell-Boltzmann distribution, the current correlations vanish [5].

In contrast, positive correlations are known to occur due to interaction in superconducting-normal hybrid structures 6], in ferromagnetic spin controlled hybrid structures 7, 8], or in normal conductors due to dynamical screening [9, 10]. However, recently Texier and Büttiker 11] predicted positive correlations in the white noise limit of purely normal conductors for a geometry [5] in which edge states are coupled to a voltage probe. The correlations change sign as the coupling to the voltage probe increases. A recent experiment by Oberholzer et al. 12 is in excellent agreement with theory. Wu and Yip 13 investigate the sign of correlations in mesoscopic $\mathrm{Y}$ structures in which one of the branches is coupled to a large resistor. It is clearly of interest to know to what extent special geometries are necessary for the observation of positive correlations in purely normal conductors.

In this Letter we point out that under very general conditions there exists a quantum mesoscopic to macroscopic crossover in purely normal conductors which manifests itself in the change of sign of current correlations. Figure 1a depicts a node of a macroscopic conductor in which one of the branches with conductance $G_{1}$ contains a source giving rise to shot noise. The two resistors on the other branches are macroscopic resistors which generate no shot noise. At the node, the electrostatic potential $U(t)$ must fluctuate, to ensure conservation of currents.
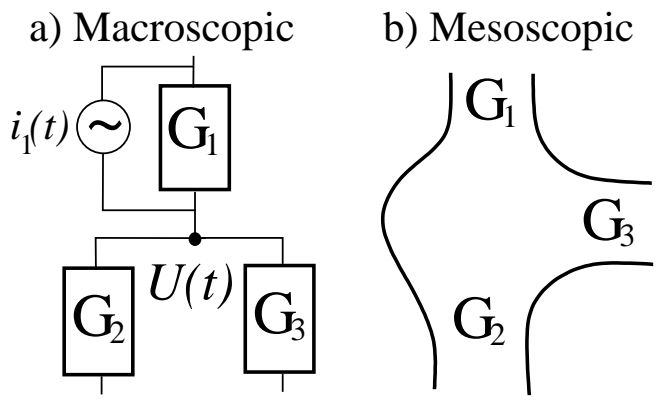

FIG. 1: Macroscopic and mesoscopic division of shot noise. The macroscopic classical circuit of Fig. 17 contains one source of noise $i_{1}(t)$. Fluctuations induced by this source give rise to a positive current correlation between currents at leads 2 and 3. Figure 10 represents a mesoscopic conductor which exhibits negative correlations. $G_{i}$ are contact conductances.

The fluctuating potential acts in a collective way on electronic carriers, correlating the currents in the branches. As a consequence such a classical circuit exhibits positive correlations. In marked contrast, in the mesoscopic conductor (see Fig. 10) carriers at different energies and in different quantum channels are uncorrelated. Accordingly a carrier exiting through a channel in one of the leads, leaves an empty state in the other out-going channels, and as consequence the current correlations are negative.

The transition from mesoscopic noise division (with negative current-correlations) to macroscopic noise division (with positive current correlations) can be investigated in a wide range of structures. It rests only on the property that correlations induced by voltage fluctuations can overwhelm correlations due to the Pauli principle.

Macroscopic versus mesoscopic noise division - The classical node (see Fig. Th) consists of three branches with conductances $G_{\alpha}$. A voltage $V$ is applied to lead 1 and the others are grounded. The conductor 1 generates shot noise with a power $p_{1}=2 \int d t\left\langle i_{1}(t) i_{1}(0)\right\rangle$. The fluctuating current through this conductor is $\Delta I_{1}(t)=$ $i_{1}(t)-G_{1} \delta U(t)$. The conductors 2 and 3 are macroscopic 
and generate no shot noise. Thus in the zero-temperature limit (which we consider from now on) the fluctuating current in these branches is $\Delta I_{i}(t)=-G_{i} \delta U(t)$ where $U(t)$ is the voltage at the node. From Kirchhoff's law it follows immediately, that the current correlation $P_{23}=\int d t\left\langle\Delta I_{2}(t) \Delta I_{3}(0)\right\rangle$ at contacts 2 and 3 is

$$
P_{23}=\frac{G_{2} G_{3}}{G_{\Sigma}^{2}} p_{1}>0
$$

where $G_{\Sigma}=\sum_{i} G_{i}$. In contrast, a mesoscopic quantum coherent conductor [1, 2] is described by scattering matrices $s_{i j}$ which give the current amplitudes in contact $i$ as a function of the incident current amplitudes in contact $j$. With the Fermi distribution in contact 1 denoted by $f_{1}$ and the Fermi distributions in contact 2 and 3 by $f_{0}$ the current correlations are [2]

$$
P_{23}=-\frac{2 e^{2}}{h} \int d E \operatorname{tr}\left[B_{32}^{\dagger} B_{23}\right]\left(f_{1}-f_{0}\right)^{2} \leq 0,
$$

where $B_{23}=s_{21} s_{13}^{\dagger}$ and the trace is over quantum channels (transverse modes). In fact the correlations of a quantum coherent conductor are negative independent of geometry and temperature, number of contacts, etc. The goal of our work is to develop a theory which connects the results of Eq. (11) and Eq. (2).

Energy conserving transport - As a generic example, it is instructive to consider next a chaotic cavity $14,15,16$ connected via contacts with conductance $G_{i}$ to reservoirs. We assume that quasi-elastic scattering is sufficiently strong such that quantum interference effects are completely washed out [17, 18, 19]. Since scattering is isotropic, the state of the cavity can be characterized by the distribution function $f_{c}(E)$. This distribution can be found from current conservation which for quasi-elastic scattering must hold at each energy. The time-averaged spectral current at contact $i$ is $\bar{I}_{i}(E)=G_{i}\left(f_{i}(E)-f_{c}(E)\right)$ and from $\sum_{i} \bar{I}_{i}(E)=0$ we find [17]:

$$
f_{c}(E)=\frac{G_{1} f_{1}(E)+\left(G_{2}+G_{3}\right) f_{0}(E)}{G_{\Sigma}} .
$$

Consider next the fluctuations away from the average current densities. Contact $i$ with $G_{i}=\left(e^{2} / h\right) \sum_{n} T_{n}^{i}$, where $T_{n}^{i}$ is the transmission probability of the $n$-th scattering channel, generates noise [1, 2, 20] with a power $p_{i}$,

$$
p_{i}=2 G_{i} \int d E\left[f_{c}\left(1-f_{c}\right)+\mathcal{F}_{i}\left(f_{i}-f_{c}\right)^{2}\right] .
$$

where $\mathcal{F}_{i} \equiv \sum_{n} T_{n}^{i}\left(1-T_{n}^{i}\right) / \sum_{n} T_{n}^{i}$ is the zerotemperature Fano factor of contact $i$. Here and in the following we have assumed energy independent transmission probabilities.

The total spectral current fluctuations $\Delta I_{i}(E, t)$ are composed of two contributions. The first contribution $i_{i}(E, t)$ is the current fluctuation of a conductor with time-independent distribution functions $f_{i}$ and $f_{c}$. A second contribution $G_{i} \delta f_{c}(E, t)$ arises from the fact that the distribution function $f_{c}$ must fluctuate to conserve current at every instant of time. Thus the total fluctuating spectral current at contact $i$ is,

$$
\Delta I_{i}(E, t)=i_{i}(E, t)-G_{i} \delta f_{c}(E, t) .
$$

Using the conservation of current fluctuations $\sum_{i} \Delta I_{i}(E, t)=0$ we obtain $\delta f_{c}=\left(i_{1}+i_{2}+i_{3}\right) / G_{\Sigma}$. Thus the $\Delta I_{i}$ can be expressed in terms of the fluctuations of $i_{i}$ alone and since the noise sources $i_{i}$ of different contacts are independent [18, 19] the current cross-correlations depend only on the auto-correlations, Eq. (44). In particular for the current-correlation $P_{23}=\int d t\left\langle\Delta I_{2}(t) \Delta I_{3}(0)\right\rangle$ at contacts 2 and 3 we find:

$$
P_{23}=\frac{G_{2} G_{3} p_{1}-G_{3}\left(G_{1}+G_{3}\right) p_{2}-G_{2}\left(G_{1}+G_{2}\right) p_{3}}{G_{\Sigma}^{2}} .
$$

We notice that the noise source of contact 1 gives a positive contribution see Eq. (11), whereas the noise sources of contacts 2 and 3 contribute with a negative sign. We remark that if all contacts contain only fully transmitting or fully reflecting modes i.e. by using a QPC at a plateau they do not produce partition noise proportional to $G_{i} \mathcal{F}_{i}$. Thus at zero temperature such a cavity exhibits noise only due to the nonzero "effective temperature" 18] $k_{B} T_{\text {eff }}=\int d E f_{c}\left(1-f_{c}\right)$ inside the cavity. The resulting correlation is negative and equal to the ensemble averaged shot noise of a quantum coherent cavity [17].

To reverse the sign of the current correlation one needs to reduce the negative contribution to Eq. (6) while keeping the positive contribution finite.

Effect of inelastic scattering - To reduce the negative contributions to the correlations in Eq. (6), we now drive the distribution function $f_{c}$ inside the cavity toward an equilibrium distribution function, thereby reducing the "effective temperature" $k_{B} T_{\text {eff }}=\int d E f_{c}\left(1-f_{c}\right)$. We allow particles in the dot to exchange energy with a voltage probe which is connected to the cavity with conductance $G_{p}$. In the following we consider contact 1 to generate shot noise with the Fano factor $\mathcal{F}_{1}$, and contacts 2 and 3 to be perfect, so that $G_{2} \mathcal{F}_{2}=0$ and $G_{3} \mathcal{F}_{3}=0$.

The voltage at the probe is found from $\bar{I}_{p}=$ $G_{p} \int d E\left(f_{p}(E)-f_{c}(E)\right)=0$, where $G_{p}=\left(e^{2} / h\right) N_{p}$ is the conductance of the contact which connects the voltage probe with the cavity. Since the distribution function in the cavity is defined from the balance of currents at each energy, it will be affected by the presence of the voltage probe,

$$
f_{c}(E)=\frac{G_{1} f_{1}(E)+G_{0} f_{0}(E)+G_{p} f_{p}(E)}{G_{\Sigma}+G_{p}} .
$$

Here, as above, $G_{\Sigma}=\sum_{i=1}^{3} G_{i}$ and $G_{0} \equiv G_{2}+G_{3}$. Substituting Eq. (7) into the equation for the current to the 
probe and performing the integration over energy we find the voltage in the probe $V_{p}=V_{1} G_{1} / G_{\Sigma}$. The distribution $f_{c}$ in the cavity has three steps (in the elastic case, Eq. (3), it has only two steps). If $G_{p}$ is small (weak inelastic scattering), then the distribution function in the cavity coincides with the elastic one and strongly deviates from an equilibrium distribution. In the limit of strong energy relaxation $f_{c}=f_{p}$ is an equilibrium distribution with the Fermi energy at $e V_{p}$. The current at the voltage probe fluctuates according to

$$
\Delta I_{p}(E, t)=i_{p}(E, t)+G_{p}\left(\delta f_{p}(E, t)-\delta f_{c}(E, t)\right),(8)
$$

which together with Eqs. (45) fully specifies the fluctuating currents.

From $\Delta I_{p}(t)=0$ we obtain $\Delta I_{i}(t)=i_{i}(t)-G_{i}\left(i_{1}(t)+\right.$ $\left.i_{2}(t)+i_{3}(t)\right) / G_{\Sigma}$ where $i_{i}(t)=\int d E i_{i}(E, t)$. Therefore the calculation proceeds as above and we obtain the current cross-correlations at contacts 2 and 3 :

$$
P_{23}=\frac{-2 G_{2} G_{3}}{G_{\Sigma}} \int d E\left[\left(f_{c}\left(1-f_{c}\right)-\frac{G_{1} \mathcal{F}_{1}}{G_{\Sigma}}\left(f_{1}-f_{c}\right)^{2}\right] .(9)\right.
$$

Using expression (77) for the distribution function and performing the integration over energy we obtain

$$
\begin{aligned}
P_{23} & =\frac{-2 e V G_{1} G_{2} G_{3}}{G_{\Sigma}\left(G_{\Sigma}+G_{p}\right)^{2}}\left[G_{0}+G_{p}+\frac{G_{p}\left(G_{0}-G_{1}\right)}{G_{\Sigma}}\right. \\
& \left.-\mathcal{F}_{1}\left(\frac{\left(G_{0}+G_{p}\right)^{2}}{G_{\Sigma}}-\frac{G_{1} G_{p}\left(2 G_{0}+G_{p}\right)}{G_{\Sigma}^{2}}\right)\right] .
\end{aligned}
$$

For $G_{p}=0$ we find the negative result for the crosscorrelations of Eq. (6), while for strong inelastic scattering $G_{p} \rightarrow \infty$ we obtain

$$
P_{23}=2 e V \mathcal{F}_{1} \frac{G_{1} G_{2} G_{3} G_{0}}{G_{\Sigma}^{3}}
$$

We see that cross-correlations are indeed positive in the case of strong inelastic scattering inside the dot.

Eq. (10) is a key result of this work. The crossover from negative to positive cross correlations of Eq. (10) are depicted in Fig. [2] for the case that all contacts have two channels. The transmission probabilities of the noise generating contact are both equal and given by $\Gamma$. The broken line and the solid line are for voltage probes with 4 and 15 channels. For small $\Gamma$ the distribution $f_{c}$ is very close to an equilibrium distribution function, and a small amount of inelastic scattering is sufficient to equilibrate the distribution. As a consequence for small $\Gamma$ the correlations are positive. As the transparency increases, the distribution function $f_{c}$ deviates strongly from the equilibrium Fermi function. The cavity is effectively "hot" and eventually the cooling provided by the voltage probe is not sufficiently strong to suppress the negative contributions to the shot noise correlation. Comparison of the curves for $N_{p}=4$ and $N_{p}=15$ shows that the range of

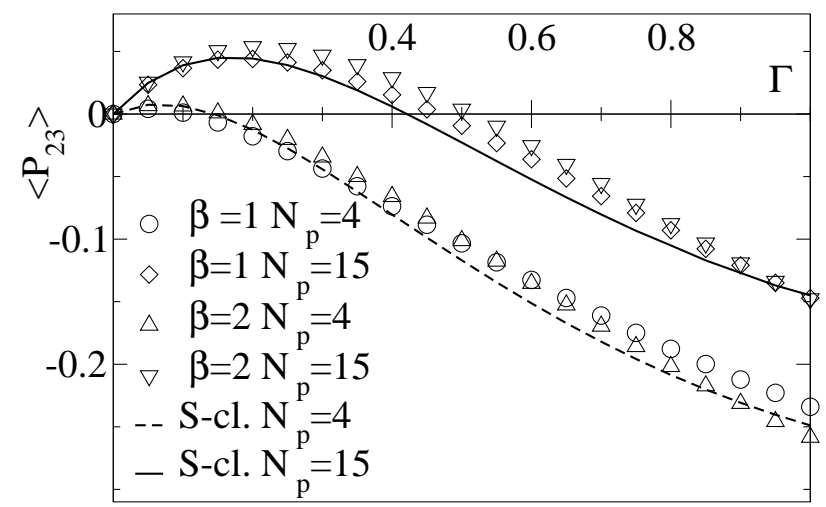

FIG. 2: Ensemble averaged current-current correlation as a function of transmission probability $\Gamma$. The full and broken lines are the analytical results of the semi-classical theory. The open symbols are from a numerical integration over an ensemble of random matrices for different symmetry classes $\beta=1,2$ and different number of channels $N_{p}$ of the voltage probe.

positive correlations is the wider the stronger the cooling of the voltage probe.

Random Matrix Theory - We next discuss the crossover for samples which are at least partially coherent. Since each sample has particular scattering properties depending on the shape of the cavity, positions of impurities, gate voltages it is interesting to consider the statistical distribution $P$ of the shot noise correlation $P_{23}$. We describe the node with a scattering matrix and as above introduce inelastic scattering with a voltage probe. For chaotic cavities, the case of interest here, the ensemble averaged noise is equal to the semi-classical result Eq. (10). In the presence of fluctuations away from the ensemble average there is therefore the interesting possibility that ensemble members might have correlations with a different sign than the ensemble averaged correlation.

Statistical properties of the transport quantities of chaotic cavities are well described by Random Matrix Theory (RMT). From our discussion we know that additional scattering at the contact 1 is essential in order to reverse sign of cross-correlations. If the quantum channels of the contacts are not fully transparent then the combined scattering matrix of the cavity and contacts can be written [21]: $S=\hat{R}-\hat{T}(\hat{1}-\hat{U} \hat{R})^{-1} \hat{U} \hat{T}$. Here $\hat{R}$ and $\hat{T}$ are reflection and transmission matrices of contacts. We chose scattering determined by $\hat{\Gamma}$ at a contact 1 and perfectly transmitting channels in all other contacts. Therefore $\hat{T}=\operatorname{diag}\{\sqrt{\hat{\Gamma}}, 1,1 \ldots\}$ and $\hat{R}=\operatorname{diag}\{\sqrt{1-\hat{\Gamma}}, 0,0 .$.$\} where \hat{\Gamma}$ is the transmission matrix of the contract 1 . $U$ is scattering matrix of the cavity itself which is distributed uniformly over the orthogonal, $\beta=1$, (unitary, $\beta=2$ ) ensemble. For simplicity we keep all transmission probabilities equal and take $\hat{\Gamma}_{n}=\Gamma$. 


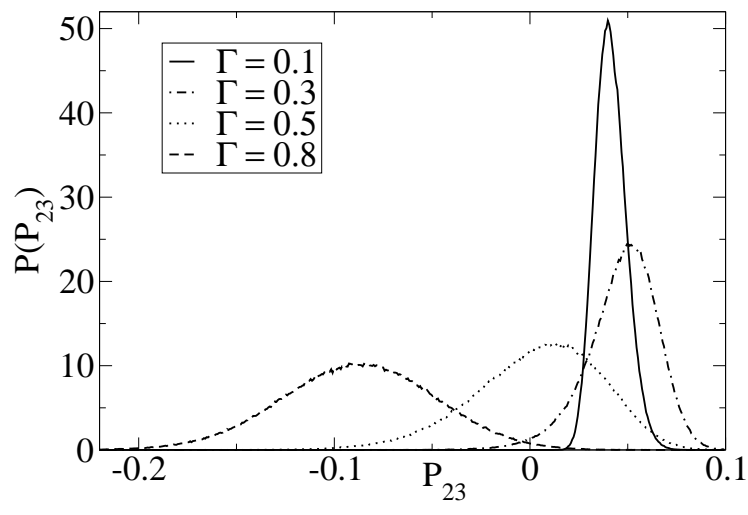

FIG. 3: Distribution of the shot noise correlation for a mesoscopic cavity. The cavity is connected via a noise generating contact with transmission $\Gamma$ per channel and two perfect contacts to reservoirs. For the case of two channels per contact and 15 channels in the voltage probe the transition from negative to positive correlations is near $\Gamma=.5$.

In the presence of a voltage probe the cross correlation $P_{23}$ can be expressed in terms of the noise correlators $p_{i j}=2 \int d t\left\langle i_{i}(t) i_{j}(0)\right\rangle$ calculated for a conductor with equilibrium distribution $f_{1}, f_{p}, f_{0}$ at the corresponding contacts. The correlation $P_{23}$ is given [10, 11, 17] by

$$
P_{23}=p_{23}-\frac{G_{2 p} p_{3 p}}{G_{p p}}-\frac{G_{3 p} p_{2 p}}{G_{p p}}+\frac{G_{2 p} G_{3 p} p_{p p}}{G_{p p}^{2}} .
$$

Here $G_{\alpha \beta}=\left(e^{2} / h\right)\left[\delta_{\alpha \beta} N_{\alpha}-\operatorname{tr}\left(S_{\beta \alpha}^{\dagger} S_{\alpha \beta}\right)\right]$ are the coherent conductances determined from the scattering matrix $S_{\alpha \beta}$.

The results of a numerical integration of Eq. (12) for the ensemble averaged cross correlation are shown in Fig. 2 for $\beta=1,2$ and $N_{p}=4,15$ and compared with the semiclassical result Eq. (10). For $\Gamma=1$ it is straightforward to evaluate 21] Eq. (12) to leading order in the number of channels for $\beta=2$,

$$
P_{23}=-\frac{4 e^{3} V}{9 h} \frac{N_{L}^{2}\left(3 N_{L}+2 N_{p}\right)}{\left(3 N_{L}+N_{p}\right)^{2}},
$$

where $N_{L}$ is the number of channels in each lead, $N_{p}$ is the number of channels in the probe. There is a perfect agreement between the semiclassical result of Eq. (10), numerical integration of Eq. (12) for $\Gamma=1$, and the analytical calculation Eq. (13).

We now obtain numerically the full statistical distribution $P$ of the current cross-correlations Eq. (12). Figure 3 shows a set of distribution functions of current crosscorrelations $P_{23}$ for different transparency $\Gamma$ of the contact 1 for $\beta=2$. For very small $\Gamma$ the distribution function is large only for positive values of the correlation. As $\Gamma$ becomes larger a tail of the distribution extends to the region of negative correlations. Eventually for large $\Gamma$ the distribution is large only for negative correlations with a tail extending to positive values of $P_{23}$.

Conclusions - Photons bunch, electrons anti-bunch! This statement is often made to explain the negative sign of current correlations in mesoscopic conductors. However, electrons are interacting entities. In particular, voltage fluctuations which accompany inelastic scattering introduce correlations which are stronger than those dictated by the Pauli principle alone. As a consequence, under a wide range of conditions, current-current correlations in normal mesoscopic conductors can be positive. We examined the crossover in detail for a range of geometries that can be subjected to experimental tests. Importantly our work demonstrates that positive correlations can in general not be used as an 'entanglement witness' since they can be due to purely classical correlations.

We thank M. Polianski, S. Oberholzer, and E.V. Sukhorukov for discussions. This work is supported by the Swiss NSF.

* Electronic address: rytchkov@physics.unige.ch

[1] Y. M. Blanter and M. Büttiker, Phys. Rep. 336, 2 (2000).

[2] M. Büttiker, Physica B 175, 199 (1991).

[3] M. Henny, et al. Science 284, 296 (1999).

[4] W. D. Oliver, et al. Science 284, 299 (1999).

[5] S. Oberholzer, et al. Physica E 6, 314 (2000).

[6] M. P. Anantram and S. Datta, Phys. Rev. B 53, 16390 (1996); T. Martin, Phys. Lett. A 220, 137 (1996); J. Torres and T. Martin, Eur. Phys. J. B 12, 319 (1999); J. Börlin et al., Phys. Rev. Lett. 88, 197001 (2002); P. Samuelsson and M. Büttiker, ibid. 89, 046601 (2002).

[7] F. Taddei and R. Fazio, Phys. Rev. B 65, 134522 (2002); D. Sanchez, et al. Phys. Rev. B 68, 214501 (2003).

[8] A. Cottet, W. Belzig, and C. Bruder Phys. Rev. Lett. 92, 206801 (2004); Phys. Rev. B 70, 115315 (2004); A. Cottet, W. Belzig, Europhys. Lett. 66, 405 (2004).

[9] A. M. Martin and M. Büttiker Phys. Rev. Lett. 84, 3386 (2000); I. Safi et al., Phys. Rev. Lett. 86, 4628 (2001); A. Crepieux et al., Phys. Rev. B 67, 205408 (2003).

[10] M. Büttiker in Quantum Noise in Mesoscopic Physics, edited by Yu. Nazarov, (Kluwer, Dordrecht, 2003), p. 3.

[11] C. Texier and M. Büttiker Phys. Rev. B 62, 7454 (2000).

[12] S. Oberholzer, E. Bieri, C. Schönenberger, M. Giovannini, and J. Faist Phys. Rev. Lett. 96, 046804 (2006)

[13] S.-T. Wu and S. Yip Phys. Rev. B 72, 153101 (2005).

[14] C.W.J. Beenakker, Rev. Mod. Phys. 69, 731 (1997).

[15] R.A. Jalabert, J.-L. Pichard, and C.W.J. Beenakker, Europhys. Lett. 27, 255 (1994)

[16] S. Oberholzer, E. V. Sukhorukov, C. Strunk, C. Schönenberger, T. Heinzel, and M. Holland, Phys. Rev. Lett. 86, 2114 (2001)

[17] S. A. van Langen and M. Büttiker, Phys. Rev. B 56, R1680 (1997).

[18] Ya. M. Blanter and E. V. Sukhorukov, Phys. Rev. Lett. 84, 1280 (2000).

[19] P. Samuelsson and M. Büttiker, Phys. Rev. B 66, 201306 (2002); M. L. Polianski, et al., Phys. Rev. B 72, 161302(R) (2005).

[20] V. A. Khlus, Sov. Phys. JETP 66, 1243 (1987); G. B. Lesovik, JETP Lett. 49, 592 (1989).

[21] P. W. Brouwer and C. W. J. Beenakker, J. Math. Phys. 37, 4904 (1996). 